\title{
INMIGRANTES Y EMIGRANTES Barrancabermeja territorio de contrastes históricos
}

\author{
Mario Andrés Alvarado Lozano \\ Especialista en Derecho Penal UNAB. Especialista en Ciencias Políticas UNAB. Psicólogo UNAB. Abogado. \\ Universidad Autónoma de Bucaramanga UNAB. Profesional en Estudios Literarios UNAB. Director del Grupo \\ de Investigaciones Facultad de Derecho, Sede Barrancabermeja-Estudios Socio-jurídicos. Docente Investigador \\ Universidad Cooperativa de Colombia UCC, Sede Barrancabermeja (Colombia). Facultad de Derecho. \\ Correo electrónico: mario.alvarado@campusucc.edu.co; alvaradoma001@hotmail.com
}

\section{Resumen}

El proyecto de investigación desarrollado tiene como objetivo central analizar la problemática socio-jurídica, familiar y personal que con mayor frecuencia presenta la población usuaria del programa de "Asistencia Jurídica Integral a Población Desplazada". Para lograr dicho objetivo la investigación se asume un diseño denominado triangulación metodológica o aproximación por métodos múltiples, dentro del cual se utilizan instrumentos de recolección de información cuantitativa como la encuesta y cuestionarios, y cualitativos como la entrevista, grupos focales y pruebas psico-diagnósticas proyectivas o subjetivas. Se espera que los resultados de la investigación arrojen luces en relación con el impacto directo que tiene sobre el proyecto de vida individual y colectiva de las personas la violación de los derechos humanos y el sometimiento a experiencias traumáticas asociadas con la vivencia del desplazamiento forzado.

Palabras Clave: Desplazamiento forzado, caracterización, violencia, conflicto armado, exclusión, educación.

\begin{abstract}
The research project aims to analyze the central socio-legal issues, family and personal that most often presents the users of the program "Legal Assistance to force displacement Integral". To achieve this research assumes a design called methodological triangulation or multiple methods approach, within which used data collection instruments and quantitative and survey questionnaires and qualitative interviews, focus groups and projective psychodiagnostic tests or subjective. It is expected that the results of the research will shed light on the direct impact on the draft individual and collective lives of people the human rights violation and submission to traumatic experiences associated with the experience of forced displacement.
\end{abstract}

Keywords: Forced displacement, characterization, violence, armed conflict, exclusion, education.

\section{Résumé}

Le projet de recherche développé a pour un objectif central analyser la problématique un partenaire juridique, familiale et personnel qu'avec une plus grande fréquence présente la population utilisatrice du programme de "Aide Juridique Intégrale à la Population Déplacée. Pour obtenir le dit objectif la recherche assume un dessin dénommé une triangulation méthodologique ou approche par des méthodes multiples, à l'intérieur qui sont utilisés des instruments de récolte d'information quantitative comme l'enquête et les questionnaires, et qualitatifs comme l'interview, les groupes focaux et tu essaies psicodiagnósticas proyectivas ou subjectives. Il s'attend que les résultats de la recherche lancent des lumières en relation avec l'impact direct qui a sur le projet de vie individuelle et collective des personnes la violation des droits de l'homme et de la soumission aux expériences traumatiques associées à l'expérience du déplacement forcé.

Mots-clés: le Déplacement forcé, la caractérisation, la violence, le conflit armé, l'exclusion, l'éducation. 

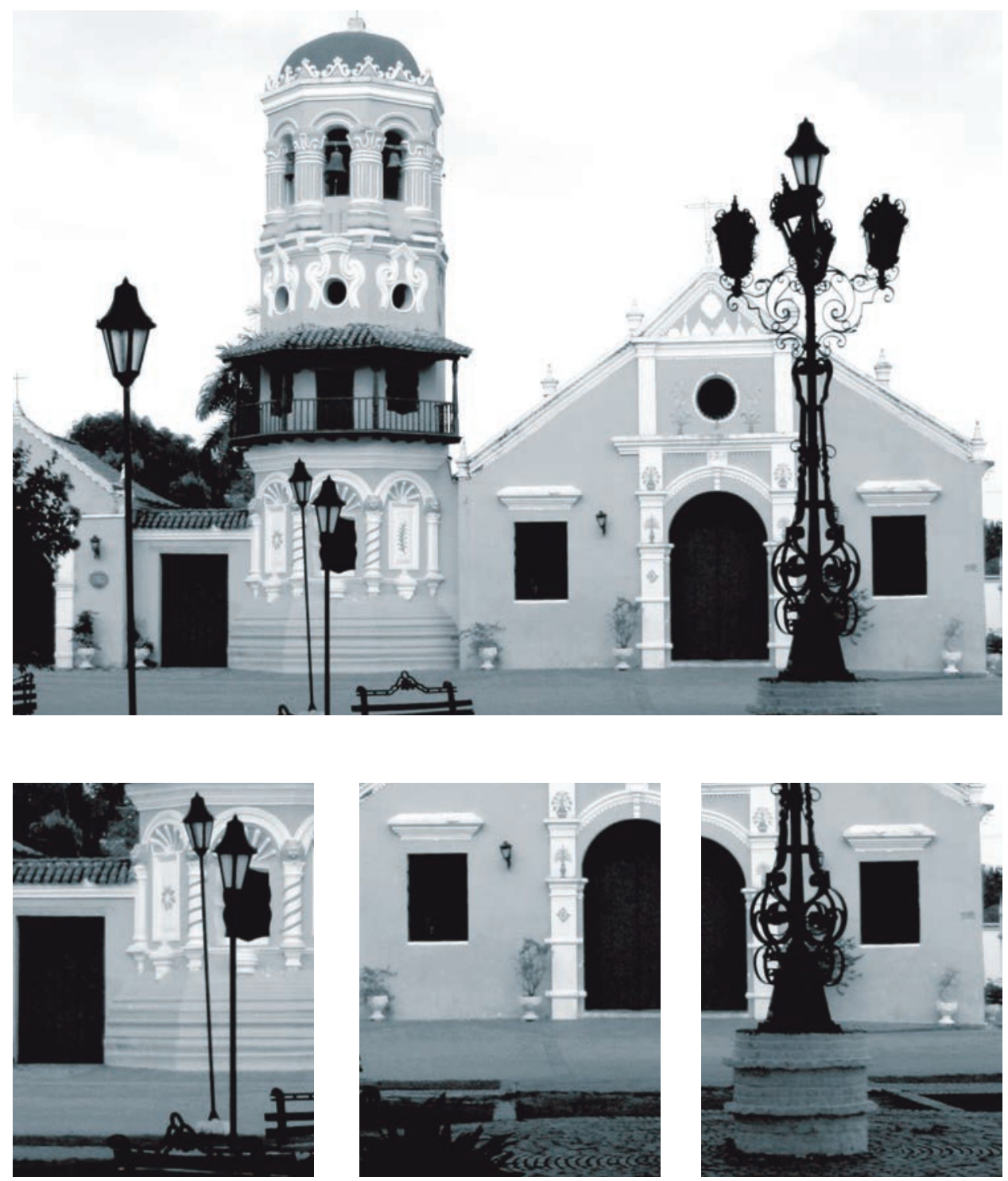

Iglesia de Santa Bárbara - Mompóx - Colombia

Martín Emilio Hernández Manrique 


\section{INMIGRANTES Y EMIGRANTES Barrancabermeja territorio de contrastes históricos*}

Mario Andrés Alvarado Lozano

Barrancabermeja existe como municipio desde 1922, sin embargo, su historia se remonta a la época precolombina; antes de la llegada de los españoles existía en este territorio la comunidad aborigen Yariguíes, quienes opusieron resistencia a los procesos de colonización implementados por los europeos. La existencia de abundante material vegetal (Caucho, Palma de Tagua, y Quina) de uso importante durante el siglo XVIII y XIX, motivó la llegada de extraños a los territorios de Barrancabermeja. Posteriormente el descubrimiento de petróleo y con ello la instalación de la Tropical Oil Company, trajo consigo inmigrantes de diversas regiones de Colombia, que veían en este lugar, la oportunidad para obtener un empleo. Así se formó la mezcla cultural que aún persiste en nuestros días.

La economía de Barrancabermeja está constituida por varias actividades entre las que se destacan la agropecuaria y en ella la producción de cacao, maíz, plátano, yuca, cítricos y una significativa población bobina, caprina y bovina. En este aparte se observa que la distribución y uso la tierra constituye una limitante para el desarrollo agrícola del municipio, si se tiene en cuenta que el $81.4 \%$ de los propietarios sólo tienen el $17.9 \%$ de la tierra, mientras que $4.1 \%$ de propietarios tienen el $45.6 \%$ de la tierra. Este nivel de concentración de la propiedad limita la posibilidad de implementación y desarrollo de la economía campesina para autoconsumo y abastecimiento local, por lo que el municipio tiene como despensa agrícola los municipios circunvecinos San Vicente de Chucurí, Yondó y Cantagallo, entre otros. El subsector pesquero también contribuye con este renglón de la economía local, en tanto que se comercializan anualmente 768 toneladas de carne de pescado de especies nativas de la región.

\footnotetext{
* El artículo es producto de la investigación: Vulnerabilidad de Derechos de población en situación de desplazamiento interno asentada en el municipio de Barrancabermeja. - Convocatoria Nacional General Comité Nacional de Investigación CONADI (UCC), Año 2010, adelantada por el autor. Grupo de Investigación Estudios Socio jurídicos UCC Sede Barrancabermeja (Colombia).
} 
Con relación al desplazamiento forzado, históricamente Barrancabermeja ha sido un centro receptor de inmigrantes, que por una $u$ otra circunstancia llegan a la ciudad. El documento del PIU, identifica tres causas de recepción a saber:

a. La economía petrolera que se ha constituido en un factor de atracción de inmigrantes a este territorio.

b. El conflicto en las regiones y zonas expulsoras de hogares.

c. Los desastres naturales, entre los que se cuenta las inundaciones del río Magdalena. Sin embargo durante la última década el factor determinante para el desplazamiento forzado y la consecuente llegada a Barrancabermeja está determinado por el conflicto armado que ha azotado a la mayoría de las regiones colombianas. Por lo que es preciso afirmar que en Barrancabermeja se observa claramente desde años atrás, la llegada de hogares que huyen de sus lugares de origen por motivo de violencia, por lo que la denominación Desplazamiento Forzado por Violencia, es un hecho real en el municipio.

En la presente investigación se realizaron 772 encuestas, en las que se caracteriza a la población en situación de desplazamiento objeto de análisis de este proyecto de la siguiente forma: La población atendida se encuentra en un rango de edad entre los 18 y 59 años, son pocas las personas mayores de 60 años, adultos mayores y menores de edad. La mayoría de la población tiene estudios de primaria, algunos con estudios de secundaria pero no culminan con el grado de bachiller, es poca la población que tiene estudios técnicos y ninguna persona atendida cuenta con estudios universitarios.

Las zonas de expulsión de víctimas de desplazamiento forzado a nivel nacional son por departamentos: Antioquia, Bolívar, Magdalena, Chocó, Cesar, Caquetá y Tolima. Y a nivel de Magdalena Medio los Departamentos de mayor expulsión son: Bolívar, Santander, Antioquia y Cesar, también se recepcionaron personas en menor proporción de los departamentos de Norte de Santander, Arauca, Magdalena, Boyacá, Sucre, Cundinamarca, Tolima, Atlántico, Meta, Caldas, Caquetá, Casanare, Cauca, Chocó, Córdoba, Guajira, Nariño, Putumayo y Valle del Cauca. Para analizar la vulnerabilidad de derechos de la población en situación de desplazamiento objeto de estudio, se realizó el estudio de las fases de la atención de acuerdo a la ley 387 de 1997, por el cual se establece la prevención, protección, atención y estabilización socioeconómica, como lo son: el Registro, la Atención de Urgencia, la Atención Humanitaria de Emergencia, la Estabilización Socioeconómica; y, adicionalmente, los derechos que se vuelven doblemente vulnerables, por la falta de garantía sistemática de las dificultades en la atención para garantizar el goce efectivo de derechos, son el Derecho a la Salud, Derecho a la Educación, Proyectos productivos, Derecho al Empleo, Derecho a la tierra y Derecho a la Vivienda. 
Tabla 1. Lugares de donde proviene la población en situación de desplazamiento

\begin{tabular}{|c|c|}
\hline $\begin{array}{c}\text { LUGAR DEL DESPLAZAMIENTO } \\
\text { DEPARTAMENTO }\end{array}$ & FRECUENCIA \\
\hline Total & 715 \\
\hline Bolívar & 237 \\
\hline Santander & 225 \\
\hline Antioquia & 164 \\
\hline Cesar & 20 \\
\hline Norte de Santander & 18 \\
\hline Arauca & 10 \\
\hline Magdalena & 9 \\
\hline Boyacá & 5 \\
\hline Sucre & 5 \\
\hline Cundinamarca & 4 \\
\hline Tolima & 3 \\
\hline Atlántico & 2 \\
\hline Meta & 2 \\
\hline Bajo Simacota & 1 \\
\hline Caldas & 1 \\
\hline Caquetá & 1 \\
\hline Casanare & 1 \\
\hline Cauca & 1 \\
\hline Choco & 1 \\
\hline Córdoba & 1 \\
\hline Guajira & 1 \\
\hline Nariño & 1 \\
\hline Putamayo & 1 \\
\hline Valle del Cauca & 1 \\
\hline
\end{tabular}

El Derecho a la salud se encuentra en primera medida garantizado al $86 \%$ de la población encuestada, el 14\% de la población no se encuentra inscrita en los servicios médicos, sin embargo, la vulnerabilidad de este derecho se presenta, con la prestación integral de los servicios, toda vez que las personas tienen la necesidad imperante de recibir atención psicológica de urgencia, médica y psicológica continuada, el embudo se dirige a la parte más baja, cuando de garantizar la prestación del servicio 
se trata, el 63\% recibió atención médica, y sólo el 47\% de la población inscrita recibió atención psicológica, menos de la mitad de la población continúa con el tratamiento de la superación de los hechos traumáticos causados por el desplazamiento forzado.

Barrancabermeja se debate entre una estrategia política militar de guerra, que desarrolla un proyecto de economía excluyente, autoritaria, marcada por la lógica del capital en un modelo agro exportador, minero extractivo y de enclave, de grandes negocios en la economía globalizada, y una apuesta a la paz, a la democracia, a la economía al servicio de la gente en armonía con la naturaleza impulsado por sectores de la ciudad de carácter civilista en los que aparece la iglesia, El Programa de Desarrollo y Paz del Magdalena Medio, las organizaciones de derechos humanos y sindicalistas entre otras.

Dos dinámicas con objetivos políticos antagónicos, que en su desarrollo las estructuras políticas, económicas, sociales de las autodefensas desmovilizadas utilizan una estratega integral de control territorial, que pasa por la coerción de los estamentos del poder público, la creación de alianzas hegemónicas y excluyentes entre ganaderos, narcotraficantes y el despliegue de redes de control político informales en áreas urbanas y rurales que al aislar y eliminar al contrario visible que era la izquierda armada del ELN y las FARC, el conflicto por el poder y el control de la economía lícita e ilícita toma una nueva dinámica en esta fase de desarrollo en la perspectiva de la precomposición del poder en Barrancabermeja y el Magdalena Medio.

En ausencia de un enemigo armado presente y de la lógica de la confrontación armada, la recomposición del poder local y regional requiere legitimar un orden social autoritario y hegemónico en una ciudad que tiene un acumulado de movimiento social, obrero y popular; recurre al uso indiscriminado de la violencia para someter y captar a las instituciones, organizaciones sociales, imponer conductas y patrones de convivencia, apropiar recursos y finanzas públicas y eliminar a quienes representen propuestas contrarias a las promovidas por la ultraderecha, en la que la población civil corre riesgos y queda expuesta a la ocurrencia de homicidios selectivos y configuración múltiple, enfrentamientos armados con interposición de población civil, destrucción de bienes civiles y el desplazamiento forzado.

En este marco de ideas el año 2006 inició con la desmovilización del Bloque Central Bolívar, que generó, expectativa en los pobladores toda vez que este actor armado ilegal se erigía como protagonista importante de la crisis humanitaria y de Derechos Humanos que padecía Barrancabermeja y la región del Magdalena Medio. Según datos de Acción Social, los motivos de desplazamiento se generaban desde la sindicación de pertenecer a organizaciones sociales defensoras de Derechos Humanos, sindicatos, organizaciones políticas de izquierda; hasta las desavenencias al interior de las autodefensas, hoy desmovilizadas, y el incumplimiento de cuotas reglamentarias en dinero de personas que utilizan el sistema de crédito fácil y no podían cancelar sus deudas en los plazos fijados. De igual manera un significativo número de jóvenes tuvieron que abandonar la ciudad por la presión de supuestos 
grupos de limpieza social quienes los sindican de ser drogadictos, ladrones o, simplemente por no corresponder a sus propósitos delincuenciales.

El desplazamiento forzado se constituye en una de las problemáticas sociales que requiere de grandes esfuerzos y exige asumir un compromiso por parte de la investigación a través de la Universidad y la sociedad civil en general. El desplazamiento interno en Colombia ha adquirido dimensiones preocupantes debido al recrudecimiento del conflicto armado en el país. Como testimonio de ello se encuentran las múltiples investigaciones y conocimientos producidos sobre el tema, los cuales se han presentado como descripciones de la problemática y constituyen un avance significativo. Pero, sin duda, se requiere de investigaciones bajo otros paradigmas que permitan comprender una dimensión más cercana al sentido real del problema.

Tal y como lo encuentra la investigación en lo referente a la categoría de efectos psicosociales, se encontró en los documentos analizados una significativa información, cuyos planteamientos centrales se dirigen a mostrar las graves consecuencias del desplazamiento y la necesidad de un abordaje interdisciplinario del fenómeno. Se evidencia, además, la urgencia que demanda la población desplazada por encontrar un nuevo rumbo para su vida y la de sus familias, por minimizar ese sentimiento de inseguridad desatado por la ausencia de futuro y por comenzar a generar estrategias a partir de las cuales las propias víctimas reconstruyan su tejido social, encuentren y construyan perspectivas de vida alcanzables con un claro sentido ético.

Las causas de desplazamiento en su orden de mayor relevancia son: amenazas generales y específicas, ataques indiscriminados, enfrentamientos armados, masacres y toma de población. Otras causas más específicas: desaparición forzada de un familiar, toma de población por parte de la guerrilla, toma de población por parte de los paramilitares, fumigaciones con glifosato, homicidio de familiar, homicidios de vecinos, miedo y reclutamiento de menores.

Para analizar la vulnerabilidad de derechos de la población en situación de desplazamiento objeto de estudio, se realizó el estudio de las fases de la atención de acuerdo a la ley 387 de 1997, por la cual se establece la prevención, protección, atención y estabilización socioeconómica, como lo son: el registro, la Atención de Urgencia, la Atención Humanitaria de Emergencia, la Estabilización Socioeconómica; y adicionalmente a ello, los derechos que se vuelven doblemente vulnerables, por la falta de garantía sistemática de las dificultades en la atención para garantizar el goce efectivo de derechos, son el Derecho a la Salud, Derecho a la Educación, Proyectos productivos, Derecho al Empleo, Derecho a la tierra y Derecho a la Vivienda. Para el registro de la población en situación de desplazamiento las personas acuden generalmente a la Personería Municipal de Barrancabermeja o al funcionario de la misma entidad que presta sus servicios en la Unidad de Atención y Orientación para población en Situación de Desplazamiento -UAO. Y también acuden en menor proporción a la Defensoría del Pueblo, a la Procuraduría no se acude porque esta entidad funciona como órgano de control para garantizar el cumplimiento de las 
fases de la atención de las diferentes instituciones vinculadas al Sistema Nacional de Atención Integral a Población Desplazada.

Las personas que declaran sobre su desplazamiento, han sufrido o han sido víctimas del mismo delito por actores, causas, en tiempos y lugares diferentes, no solamente de uno sino de dos y tres desplazamientos. Las personas que fueron víctimas varias veces, solamente declaran un solo hecho y las entidades del ministerio público no registran la magnitud de los sucesos, o la frecuencia con que le ocurre a la población que ha sido registrada, genera una vulnerabilidad en el derecho a la justicia, porque los anteriores hechos quedan totalmente en la impunidad.

El registro no se genera de forma automática con sólo declarar, por parte de Acción Social corresponde la valoración de la declaración y notificar a la persona en situación de desplazamiento la resolución de inclusión o no en el mismo. De la población declarante el $37 \%$ no fue incluida en el registro inmediatamente, el $63 \%$ fue incluido con solo la declaración, el $95 \%$ de la población no incluida inicialmente con la declaración, fue incluida con el agotamiento de la vía gubernativa, es decir, con interponer los recursos de reposición y en subsidio de apelación, el $5 \%$ de esa población fue incluida con los mecanismos de exigibilidad de derechos fundamentales, derechos de petición y acciones de tutelas. Las personas que han tenido más de dos desplazamientos, se les pregunta si han sido ofrecidas las opciones de reubicación o retorno, pero en pocos casos, casi que excepcionalmente se le orienta a la víctima declarante que puede optar por estas opciones de vida.

La encuesta contempló la opciones de retorno, reubicación o reasentamiento, a lo que las personas responden preferir ser reasentadas, quedarse en el lugar donde lo recibieron y declaró su desplazamiento para continuar con su vida, otro número considerable aceptaría la opción de hacer parte de procesos de reubicación, sin embargo la opción que menos se prefiere por falta de garantías de seguridad y de no repetición de los hechos que ocasionaron el desplazamiento forzado es la de retornar o volver a las tierras y lugares abandonados por la violencia.

Barrancabermeja ha desarrollado a nivel interinstitucional y coordinado desde la Alcaldía Municipal, específicamente de la Unidad de Atención y Orientación UAO, para garantizar el proceso de Atención de Urgencia, una ruta que permite a las personas garantizar sus necesidades básicas mientras se desarrolla el procedimiento de inclusión en el RUPD. El convenio suscrito entre la Alcaldía Municipal y el Servicio Jesuita a Refugiados garantiza el alojamiento y la alimentación de las familias en situación de desplazamiento, allí se le brinda atención médica y psicológica con la articulación de los programas del ICBF y la Secretaría de Salud. La mitad de la población encuestada aproximadamente ha recibido ayuda inmediata, no se podría afirmar que la otra mitad, no la ha recibido por falta de ofrecimiento institucional, dado que los tiempos de declaración y de ocurrencia del delito de desplazamiento forzado pueden variar, y consecuencia de ello, lo innecesario de brindar una atención de urgencia, por no encontrarse la persona en inminente necesidad o extrema urgencia. 
De la población que afirmó haber recibido ayuda inmediata, el $40 \%$ aproximadamente, dijo haber recibido alojamiento transitorio en casas, albergues o dinero para el alojamiento, la variación de estas opciones obedece a que con el pasar de los años varían las estrategias de atención por parte de las instituciones del SNAIPD para solucionar las situaciones de urgencias de la población en situación de desplazamiento. Más del $70 \%$ de la población que recibió alojamiento transitorio, fue dotada con los kits de aseo, cocina, habitad y alimentación.

La Coordinación interinstitucional se fractura en la fase de inclusión en el RUPD, toda vez que Acción Social, dilata los términos de valoración de la declaración y, a su vez, de la expedición de la resolución de inclusión o no inclusión, las personas que son atendidas en el Albergue tienen que permanecer un mes o más en espera de esta respuesta, y en ocasiones, corresponde al operador del mismo solicitar el desalojo voluntario de las familias, que en esas condiciones desbordan la capacidad presupuestal de la Alcaldía Municipal para su manutención y sostenimiento durante varios meses. Las personas en situación de desplazamiento que se encuentran en el Albergue deben desplazarse a las oficinas del Ministerio Público (cuando no son desplazamientos masivos), y a las instalaciones de la UAO para cubrir la ruta de inclusión en el Registro y para la Atención Humanitaria de Emergencia, esto expone a las personas a un mayor riesgo de seguridad y las distancias son considerables para acudir a dichas instituciones.

El $78 \%$ de la población encuestada recibió la atención humanitaria de emergencia, no se especifica en este análisis si fue en especie o en dinero, Acción Social reporta, para el tema del pronunciamiento de la Corte Constitucional, que sostuvo en la sentencia T-285 de 2008, que la prórroga de la atención humanitaria de emergencia, debe realizarse hasta que el afectado esté en condiciones de asumir su auto sostenimiento, que se han visto colapsados los recursos de esta fase con las constantes solicitudes de prórroga de la atención, sin embargo, el análisis reporta que solamente el 7\% de la población ha recibido la prórroga de la atención humanitaria de emergencia. La entrega de la Atención Humanitaria de Emergencia se ha convertido en una de las prioridades de Acción Social en Barrancabermeja, las personas han reclamado la Atención Humanitaria de Emergencia, pero esto no obedece a la atención sistemática, coordinada, planeada y progresiva para que las personas garanticen durante el tiempo estipulado por la ley sus necesidades básicas como alimentación y alojamiento, puesto que, se hacen jornadas masivas de asignación de la Atención Humanitaria de Emergencia, dónde a las personas les entregan un monto (diferenciado por tipos: A, B o C), y aparece en el sistema que se le ha garantizado esta fase de la atención, razón por la cual, las personas recurren posteriormente a la solicitud de la prórroga de la Atención Humanitaria de emergencia.

El 10\% de la población indagada responde haber recibido dinero o implementos para desarrollar su propio proyecto productivo. Ha sido este recurso para proyectos de emprendimiento, palma, ganadería, pollos, o actividades informales como la elaboración artesanal de productos para la venta, sandalias, correas, bolsos, muñecos, 
o el surtimiento de mercancía para la reventa en tiendas, almacenes y en sus casas. Existe una ruptura entre la coordinación de las instituciones del SNAIPD local, y de este a su vez con el departamental y nacional para el cumplimiento de la atención de esta fase, y que por consiguiente todo el peso de la atención a la población en situación de desplazamiento se devuelve a las primeras fases de atención convirtiéndose en una barrera para la superación del estado de cosas inconstitucional.

Los mecanismos, rutas y procedimientos creados por Acción Social solo obedecen a su propia dinámica interna con la entrega de Plan Semilla o Proyecto Productivo, en el municipio se ha avanzado en esta fase por los esfuerzos institucionales del SNAIPD local en garantizar de forma individual cada uno de los derechos de la población en situación de desplazamiento de acuerdo a su competencia. El 2\% de las personas que han implementado algún proyecto productivo ha buscado la forma de adquirir un crédito para hacer crecer el proyecto o el negocio, sin dar cuenta que los mismos prosperen o fracasen.

Los empleos otorgados, han sido en la zona urbana, cosa que nos permite indicar, que el proceso de adaptabilidad de las personas es casi nulo, debido a que las personas no cuentan con el estudio y la experticia de los empleos que requieren para trabajar en la zona urbana. El empleo, se ha considerado una de las principales garantías constitucionales para poder decir que se establece socioeconómicamente a la persona en situación de desplazamiento. Sin embargo, la Alcaldía Municipal ha generado políticas públicas para contrarrestar el tema de desempleo en la población en situación de desplazamiento, pero que por la gravedad de la situación se han convertido en medidas incipientes, insistiendo, en que son en vano los alcances municipales toda vez que este, no ha contado con el respaldo del nivel nacional y departamental.

Los empleos otorgados a la población en situación de desplazamiento en la zona urbana, obedece a subempleos, o a empleos que no requieren un conocimiento técnico o profesional, que pueden en caso ser empleos informales, sin las garantías y prestaciones sociales que denota la empleabilidad en un manejo de la política pública de empleo para la población en situación de desplazamiento, y poniendo en evidencia, que se vulnera el derecho al trabajo de la población en situación víctima de este delito. Hay un total de 1.856 hectáreas abandonadas por el delito del desplazamiento forzado del número de personas encuestadas. Que el 49\% de la población dice ser propietaria de las tierras, el $30 \%$ tenedor y el $21 \%$ poseedor, el vínculo mayor con el inmueble es el propietario con justo título. Se observa, que hay una distribución equitativa de la tierra, es decir, la mayoría de los propietarios, son dueños de pequeñas extensiones de tierra, de parcelas con poca magnitud de terreno, pero que sumadas, dan cuenta de la gran cantidad de población rural víctima del desplazamiento forzado. 
Tabla 2. Frecuencia de los empleos realizados por la población en situación de desplazamiento en la zona urbana

\begin{tabular}{|c|c|}
\hline EN QUE REALIZÓ EL EMPLEO & FRECUENCIA \\
\hline Albañilería & 2 \\
\hline Cosiendo forros para computador & 2 \\
\hline Empleada doméstica & 2 \\
\hline Jardinería & 2 \\
\hline Impulsadora & 2 \\
\hline Lavando y planchando & 2 \\
\hline Yambal & 2 \\
\hline Total & 14 \\
\hline
\end{tabular}

Las personas en situación de desplazamiento han perdido y dejado abandonadas las tierras o bienes inmuebles en el área rural y urbana, pero esta situación ha quedado de lado como componente de reparación toda vez, que el Desplazamiento Forzado se declara para efectos meramente administrativos, pero no para la protección del derecho que involucra el área penal y policivo. El 89\% de la población no ha solicitado subsidio de vivienda, iniciando con el primer paso de la ruta que garantizaría este derecho. $11 \%$ de los encuestados dice haber solicitado el subsidio. Del 11\% que solicitó el subsidio el $67 \%$ ha recibido el subsidio de vivienda, el 33\% de esa población no lo recibe. Hay que anotar que el contexto económico en que vive la ciudad de Barrancabermeja, por el auge petrolero y la industria, ha generado que los costos en el nivel de vida se haya aumentando, no queriendo decir que sea directamente proporcional con el desarrollo social, sin embargo, ha creado un alza incontrolable de los precios de las viviendas que imposibilita a la administración municipal demostrar los esfuerzos realizados en este tema.

\section{Lectura de resultados}

La lectura de los resultados encontrados, deja observar en esta investigación, la situación sobre el tema de desplazamiento forzado en Colombia, a partir de los múltiples argumentos descriptivos obtenidos a través de las fuentes documentales analizadas. Si bien se constituyó en un esfuerzo importante, es preciso darle continuidad, bien sea a partir de la comprensión e interpretación de cada una de las categorías de análisis establecidas o bien comenzando con otras nuevas, bajo el entendido que Barrancabermeja históricamente se he configurado como territorio de multiculturalidad, de inmigraciones y emigraciones. 
La lectura permite revisar los siguientes resultados: se realizaron 772 encuestas, en las que se caracteriza a la población en situación de desplazamiento objeto de análisis de este proyecto de la siguiente forma: La población atendida se encuentra en un rango de edad entre los 18 y 59 años, son pocas las personas mayores de 60 años, adultos mayores y menores de edad. La mayoría de la población solamente tiene estudios de primaria, algunos con estudios de secundaria pero no culminan con el grado de bachiller, es poca la población que tiene estudios técnicos y ninguna persona atendida cuenta con estudios universitarios. Las zonas de expulsión de víctimas de desplazamiento forzado a nivel nacional son por departamentos: Antioquia, Bolívar, Magdalena, Chocó, Cesar, Caquetá y Tolima. Y a nivel de Magdalena medio los Departamentos de mayor expulsión son: Bolívar, Santander, Antioquia y Cesar, también se recepcionaron personas en menor proporción de los departamentos de Norte de Santander, Arauca, Magdalena, Boyacá, Sucre, Cundinamarca, Tolima, atlántico, meta, Caldas, Caquetá, Casanare, Cauca, Chocó, Córdoba, Guajira, Nariño, Putumayo y Valle del Cauca.

Los municipios de mayor expulsión por Departamento, vienen siendo las cabeceras municipales como puertos fluviales, pero no son expulsoras en mayor ni menor porcentaje las capitales de cada departamento. Así, en el departamento de Bolívar, son expulsores en mayor proporción, Cantagallo y San Pablo, pertenecientes al Sur de Bolívar, en Santander Barrancabermeja y Simacota, y en el Departamento de Antioquia Yondó y Puerto Berrio. Las causas de desplazamientos en su orden de mayor relevancia son: Amenazas, generales y específicas, ataques indiscriminados, enfrentamientos armados, masacres y toma de población. Otras causas más específicas: Desaparición forzada de un familiar, toma de población por parte de la guerrilla, toma de población por parte de los paramilitares, fumigaciones con glifosato, homicidio de familiar, homicidios de vecinos, miedo y reclutamiento a menores.

Hay un total de 1.856 hectáreas abandonadas por el delito del desplazamiento forzado del número de personas encuestadas. El 49\% de la población dice ser propietaria de las tierras, el $30 \%$ tenedor y el $21 \%$ poseedor, siendo el vínculo mayor con el inmueble el propietario con justo título. Se observa, que hay una distribución equitativa de la tierra, es decir, la mayoría de los propietarios, son dueños de pequeñas extensiones de tierra, de parcelas con poca magnitud de terreno, pero que sumadas, dan cuenta de la gran cantidad de población rural víctima del desplazamiento forzado. Las personas en situación de desplazamiento han perdido y dejado abandonadas las tierras o bienes inmuebles en el área rural y urbana, pero esta situación ha quedado de lado como componente de reparación toda vez, que el Desplazamiento Forzado se declara para efectos meramente administrativos, pero no para la protección del derecho que involucra el área penal y policivo.

El $89 \%$ de la población no ha solicitado subsidio de vivienda, iniciando con el primer paso de la ruta que garantizaría este derecho, 11\% de los encuestados dice haber solicitado el subsidio. Del $11 \%$ que solicitó el subsidio el $67 \%$ ha recibido el 
subsidio de vivienda, el 33\% de esa población no lo recibe. Hay que anotar que el contexto económico en que vive la ciudad de Barrancabermeja, por el auge petrolero y la industria, ha generado que los costos en el nivel de vida se haya aumentando, no queriendo decir que sea directamente proporcional con el desarrollo social, sin embargo, ha creado un alza incontrolable de los precios de las viviendas, imposibilitando a la administración municipal demostrar los esfuerzos realizados en este tema.

\section{CONCLUSIONES}

Las zonas de expulsión de víctimas de desplazamiento forzado a nivel nacional son por departamentos: Antioquia, Bolívar, Magdalena, Chocó, Cesar, Caquetá y Tolima. Y a nivel de Magdalena medio los Departamentos de mayor expulsión son: Bolívar, Santander, Antioquia y Cesar, también se recepcionaron personas en menor proporción de los departamentos de Norte de Santander, Arauca, Magdalena, Boyacá, Sucre, Cundinamarca, Tolima, atlántico, meta, Caldas, Caquetá, Casanare, Cauca, Chocó, Córdoba, Guajira, Nariño, Putumayo y Valle del Cauca. Los municipios de mayor expulsión por Departamento, vienen siendo las cabeceras municipales como puertos fluviales, pero no son expulsoras en mayor ni menor porcentaje las capitales de cada departamento. Así, en el departamento de Bolívar, son expulsores en mayor proporción, Cantagallo y San Pablo, pertenecientes al Sur de Bolívar, en Santander Barrancabermeja y Simacota, y en el Departamento de Antioquia Yondó y Puerto Berrío.

El municipio de Barrancabermeja ha venido haciendo esfuerzos institucionales desde el año 2006 para implementar políticas públicas de atención a la Población en situación de desplazamiento. No obstante, se presentan dificultades en la coordinación de los comités encargados de llevar adelante dichas políticas. Dentro de las dificultades más frecuentes se encuentran, el fortalecimiento de los canales de comunicación entre los comités y entes encargados de brindar asistencia a la población, la falta de organización de información recogida de dicha población, la demora para tomar decisiones fundamentales que impactan a la población en situación de desplazamiento y demás.

El problema de la coordinación en la asistencia de la PSD no es un problema menor, ya que finalmente se sabe que sin una política pública seria, será muy difícil que se puedan superar las condiciones a las que este fenómeno somete a sus afectados. Además se ha evidenciado que existen contradicciones desde la esfera de lo político en la asistencia y tratamiento a esta población desde la implementación de las políticas públicas, para contrarrestar estos efectos desde el ámbito nacional se han realizado esfuerzos para convertir las políticas de gobierno en políticas de Estado, y así poder garantizar la continuidad y perfeccionamiento de las políticas ideadas para combatir y superar este fenómeno gradualmente, con eficacia y celeridad. 
Igualmente se evidencio, que los entes involucrados en el afrontamiento del fenómeno de desplazamiento forzado, a pesar del interés demostrado, tienen grandes dificultades en el tema de la caracterización de la PSD, lo que trae retrasos en la capacidad de respuesta al fenómeno, esto en parte sigue siendo resultado de la inexistencia de un enfoque común para el tratamiento del fenómeno y la escasa coordinación del orden nacional-municipal en esta problemática, falta control desde lo nacional y un compromiso más sistemático y decidido desde lo municipal, para cumplir con los compromisos de atención integral a la PSD en el municipio de Barrancabermeja. Aunque hay que apuntar, que desde el año 2009 se vienen realizando sendos esfuerzos para superar estas condiciones que dificultan la aceleración del proceso de superación del fenómeno que ha padecido la región del magdalena medio con más intensidad desde hace por lo menos dos décadas.

\section{REFERENCIAS}

Archila, M. (1991). Cultura e identidad obrera. Bogotá, CINEP.

CODHES. (2000). Guerra del desplazamiento y pobreza. En CODHES Informa. Boletín de la Consultoría para los Derechos Humanos y Desplazamientos a Marchas Forzadas. 30 (agosto). CODHES. Bogotá.

Conferencia Episcopal de Colombia. (1995). Boletín Trimestral sobre el Desplazamiento Forzado en Colombia. 8 (septiembre-diciembre). Editorial Kimpres. Bogotá.

Contreras, Daniel, Olivares, Alejandro. Artículo de opinión: "Democracia en América Latina" en http://aolivares.blogspot.com/2005_08_01_aolivares_archive

Departamento Nacional de Planeación-DNP, Colombia. (1995). Programa Nacional de Atención Integral a la Población Desplazada por la Violencia. Documento Conpes 2804. Ministerio del Interior-Consejería Presidencial para los Derechos Humanos. Bogotá.

Diócesis de Barrancabermeja. (2001). La luz de esperanza, Barrancabermeja.

Falla, U., Chávez Y. y Molano, G. (2001). Desplazamiento forzado en Colombia: Estado del arte y una experiencia en la Unidad de Atención Integral a esta población. Informe final de la investigación. Universidad Colegio Mayor de Cundinamarca. Bogotá.

Franco, A. (1998). Los desplazamientos internos en Colombia. Una conceptualización política para el logro de soluciones a largo plazo. En Colombia Internacional. Publicación del Centro de Estudios Internacionales de la Universidad de los Andes. 42 (abril-junio): 5-27. Universidad de los Andes. Bogotá.

González, F. (1997). Aproximación a la configuración Política Colombiana, en Para Leer la Política, Tomo I, CINEP, Santafé de Bogotá. 
Guillen, F. (1986). La Regeneración” Primer Frente Nacional, Carlos Valencia Editores, Bogotá. Págs. 15-70 los partidos políticos.

Grupo Organizaciones de Apoyo a Desplazados. (2001). Éxodo. Boletín de Desplazamiento Interno en Colombia. Editorial GAD. Bogotá.

López, O. (2000). Investigación y trabajo psicosocial con familias víctimas de la guerra. Revista Colombiana de Trabajo Social. Consejo Nacional para la Educación en Trabajo Social. Serie 14. CONETS. Cali.

Ministerio de Salud-Fundación Educativa Amor (s.f.). Presentación propuesta de adaptación psicosocial a familias desplazadas de campo por la violencia. CINEP. Bogotá. Naciones Unidas-Defensoría del Pueblo, Colombia. Principios rectores de los desplazamientos internos. Naciones Unidas-Defensoría del Pueblo. Bogotá.

Organización Panamericana de la Salud-CODHES-UNICEF. (1999). Un país que huye. Desplazamiento y violencia: una nación fragmentada. Ed. Guadalupe. Bogotá.

Osorio, F. (1993). La violencia del silencio: desplazados del campo a la ciudad. CODHES -Pontificia Universidad Javeriana. Bogotá.

Pastrana, A, López, C y Solimano, A. (s.f.). Ensayo sobre paz y desarrollo y otros. Tercer Mundo Editores. Bogotá.

Pérez, D. (1995). El desplazamiento forzado en Colombia: ruptura del tejido social y el proyecto vital». En Su Defensor. Periódico de la Defensoría del Pueblo para la divulgación de los derechos humanos. 21. Defensoría del Pueblo. Bogotá.

PNUD. (2004). "El desarrollo de la democracia en América Latina". En la Democracia en América Latina. Hacia una democracia de ciudadanas y ciudadanos, PNUD.

Vargas, A. (1998). Las Fuerzas Armadas en el Conflicto Colombiano. Antecedentes y perspectivas, Intermedio Editores, Bogotá. Capítulo 2. 